\title{
Variation on a theme of MacDonald
}

\section{R. Brussee ${ }^{1}$, G.J. Heckman ${ }^{2}$, and E.M. Opdam ${ }^{1}$}

\begin{abstract}
${ }^{1}$ Mathematisch Instituut, Rijksuniversiteit Leiden, Postbus 9512, NL-2300 RA Leiden, The Netherlands

${ }^{2}$ Mathematisch Institut, Katholicke Universiteit Nijmegen, Toernooiveld, NL-6525 Nijmegen, The Netherlands
\end{abstract}

Received in final form January 22, 1991

1 Introduction.

2 Conditions for integrability . . . . . . . . . . . . 3

3 The case $R$ of type $B C_{n}$. . . . . . . . . . . . . . . . . . . . . . . . . . . . . . 4

4 From compact imaginary to non-compact real . . . . . . . . . . . 5

5 Evaluation of the non-compact integral . . . . . . . . . . . . . . . 6

6 Final remarks . . . . . . . . . . . . . . . . . . . . . . . . . 9

\section{Introduction}

The classical Euler $B$-integral

$$
B(x, y):=\int_{0}^{1} t^{x-1}(1-t)^{y-1} d t=\frac{\Gamma(x) \Gamma(y)}{\Gamma(x+y)}(\operatorname{Re}(x)>0, \operatorname{Re}(y)>0) .
$$

can be rewritten (by the substitution $\left.t=\frac{a}{a+1}\right)$ as

$$
B(x, y)=\int_{0}^{\infty} a^{x-1}(1+a)^{-x-y} d a .
$$

A multivariable generalization of (1.1) due to Selberg [S] says that for

$$
\operatorname{Re}(x)>0, \quad \operatorname{Re}(y)>0, \quad \operatorname{Re}(z)>-\min \left\{\frac{1}{n}, \frac{\operatorname{Re}(x)}{n-1}, \frac{\operatorname{Re}(y)}{n-1}\right\}
$$

we have

$$
\begin{aligned}
B_{n}(x, y, z) & :=\int_{0}^{1} \ldots \int_{0}^{1}\left(t_{1} \ldots t_{n}\right)^{x-1}\left\{\left(1-t_{1}\right) \ldots\left(1-t_{n}\right)\right\}^{y-1}|\Delta(t)|^{2 z} d t_{1} \ldots d t_{n} \\
& =\prod_{j=1}^{n} \frac{\Gamma(1+j z) \Gamma(x+(j-1) z) \Gamma(y+(j-1) z)}{\Gamma(1+z) \Gamma(x+y+(n+j-2) z)}
\end{aligned}
$$

where $\Delta(t)$ denotes the discriminant

$$
\Delta(t)=\Delta\left(t_{1}, \ldots, t_{n}\right)=\prod_{i<j}\left(t_{i}-t_{j}\right) .
$$


The same substitution $t_{i}=\frac{a_{i}}{a_{i}+1}$ carries Selberg's integral over into the formula

$$
\begin{gathered}
B_{n}(x, y, z)=\int_{0}^{\infty} \ldots \int_{0}^{\infty}\left(a_{1} \ldots a_{n}\right)^{x-1} \cdot\left\{\left(1+a_{1}\right) \ldots\left(1+a_{n}\right)\right\}^{-x-y-2 z(n-1)} \\
\cdot|\Delta(a)|^{2 z} d a_{1} \ldots d a_{n} .
\end{gathered}
$$

Suppose $\mathfrak{a}$ is a Euclidean space with inner product $(\cdot, \cdot)$. The dual space $\mathfrak{a}^{*}=\operatorname{Hom}(\mathfrak{a}, \mathbb{R})$ inherits a natural inner product from $\mathfrak{a}$, which we again denote by $(\cdot, \cdot)$. Let $R \subset \mathfrak{a}^{*}$ be an irreducible reduced root system [B]. The coroot lattice $Q^{\vee} \subset a$ is the lattice generated by the dual root system $R^{\vee}=\left\{\alpha^{\vee}\right.$ $\left.:=\frac{2(\alpha, \cdot)}{(\alpha, \alpha)} \in \mathfrak{a} ; \alpha \in R\right\}$, and the weight lattice $P=\left\{\lambda \in \mathfrak{a}^{*} ;\left(\lambda, \alpha^{\vee}\right) \in \mathbb{Z} \forall \alpha \in R\right\}$ is the lattice in $\mathfrak{a}^{*}$ dual to $Q^{\vee}$. The Weyl denominator is by definition

$$
\Delta:=\prod_{\alpha \in \mathbf{R}_{+}}\left(e^{\frac{1}{2} \alpha}-e^{-\frac{1}{2} x}\right) \in \mathbb{Z}[P]
$$

where $R_{+} \subset R$ is a set of positive roots.

Let $T$ be the compact torus with (abelian) Lie algebra $t:=\sqrt{-1} \mathfrak{a}$ and unit lattice $2 \pi \sqrt{-1} Q^{\vee}$, so that $\Delta(t)$ for $t \in T$ can be considered as a Fourier polynomial on $T$ with integral coefficients. If $2=d_{1} \leqq d_{2} \leqq \ldots \leqq d_{n}=h$ denote the degrees of $R$ ( $h$ being the Coxeter number), and $d t$ the normalized Haar measure on $T$ then

$$
\int_{T}|\Delta(t)|^{2 k} d t=\prod_{j=1}^{n}\left(\begin{array}{c}
k d_{j} \\
k
\end{array}\right) \quad \text { for } k>-h^{-1}
$$

This formula was conjectured by Macdonald [M2]. In fact Macdonald conjectured a more general formula (allowing the root system to be possibly nonreduced, and different labels for roots of different lengths), which for the root system of type $B C_{n}$ was equivalent with Selberg's integral (1.3). For the root system of type $A_{n}$ formula (1.6) was conjectured by Dyson [D], and proved by Gunson [G] and Wilson [Wi] (and unpublished by Selberg [S, p. 212]).

A uniform proof of (1.6) for all root systems was given by Opdam as an application of the calculus of multivariable hypergeometric shift operators [O3]. The original proof of the existence of these hypergeometric shift operators depended on transcendental arguments [O2], but now this can also be understood by elementary means [H2].

Let $A$ be the connected simply connected Lie group with Lie algebra a (the Lie bracket on $\mathfrak{a}$ being trivial), so that $\exp : \mathfrak{a} \rightarrow A$ is a diffeomorphism. Normalize the Haar measure $d a$ on $A$, such that $\int_{A / \exp \left(2 \pi Q^{\nu}\right)} d a=1$. This is the canonical measure on $A$ obtained from the normalized Haar measure $d t$ on $T$ by analytic continuation. The Weyl denominator $\Delta(a)$ for $a \in A$ can be considered as an exponential polynomial on $A$. 
Theorem 1.1 For $-h^{-1}<\operatorname{Re}(k)<0$ we have

$$
\int_{A}|\Delta(a)|^{2 k} d a=\prod_{j=1}^{n} \frac{1}{2 \sin \left(\pi k\left(1-d_{j}\right)\right)} \cdot \prod_{j=1}^{n}\left(\begin{array}{c}
k d_{j} \\
k
\end{array}\right)
$$

In fact we will prove a more general formula for $R$ possibly non-reduced and different labels for roots of different lengths. For $R$ of type $B C_{n}$ this formula is equivalent with Selberg's integral (1.4) as will be shown in Sect. 3. In Sect. 4 we will show how the compact imaginary and non-compact real integrations can be related with the help of Stokes theorem. However for this we have to replace the integrals with measures by the corresponding integrals with differential forms. In Sect. 5 we finish the proof of (1.7) by connecting the integrals for measures and differential forms using the Poincare series for both the finite and the affine Weyl group.

\section{Conditions for integrability}

Let $a$ be a Euclidean space of dimension $n$, and $R \subset \mathrm{a}^{*}$ a possibly non-reduced root system with Weyl group $W$. Let $R_{+} \subset R$ be a set of positive roots, and $\mathfrak{a}_{+} \subset \mathfrak{a}$ the corresponding positive chamber. Write $\mathfrak{a}_{c}=\mathbb{C} \otimes_{\mathbb{R}} \mathfrak{a}$ for the complexification of $\mathfrak{a}$, and $\mathfrak{a}_{\mathfrak{c}}^{\text {reg }}=\left\{X \in \mathfrak{a}_{c} ; \alpha(X) \neq 0 \forall \alpha \in R\right\}$ for the regular points in $\mathfrak{a}_{\boldsymbol{c}}$. Suppose we have given $k_{\alpha} \in \mathbb{C}$ for $\alpha \in R$, such that $k_{w \alpha}=k_{a} \forall w \in W, \forall \alpha \in R$.

Consider the functions on $\mathfrak{a}_{c}^{\text {reg }}$ defined by

$$
\begin{aligned}
& \delta(k, X)=\prod_{\alpha \in R_{+}}\left(e^{\frac{1}{2} \alpha(X)}-e^{-\frac{1}{2} \alpha(X)}\right)^{2 k_{\alpha}} \\
& \mu(k, X)=\prod_{x \in \boldsymbol{R}_{+}}\left|e^{\frac{1}{2} \alpha(X)}-e^{-\frac{1}{2} \alpha(X)}\right|^{2 k_{\alpha}}
\end{aligned}
$$

Here $\delta(k, \cdot)$ is the multi-valued analytic continuation of the single-valued analytic function on $\mathfrak{a}_{c,+}=\mathfrak{a}_{+} \oplus \sqrt{-1} \mathfrak{a}$ for which $\delta(k, X)=\mu(k, X)$ for $X \in \mathfrak{a}_{+}$. Note that $\mu(k, X)=|\delta(k, X)|$ if $k_{\alpha} \in \mathbb{R} \forall \alpha \in R$.

For $X \in \mathfrak{a}_{c}$ we put

$$
R_{X}=\{\alpha \in R ; \alpha(X) \in 2 \pi \sqrt{-1} \mathbb{Z}\},
$$

whence $R_{X} \subset R$ is a root subsystem.

Proposition 2.1 Write $\rho(k):=\frac{1}{2} \sum_{\alpha \in R_{+}} k_{\alpha} \alpha=\sum_{j=1}^{n} l_{j} \alpha_{j}$ with $\left\{\alpha_{1}, \ldots, \alpha_{n}\right\} \subset R_{+}$the set of
simple roots.

a) If $\operatorname{Re}\left(\sum_{\alpha \in R_{X}} k_{\alpha}\right)+r k\left(R_{X}\right)>0 \forall X \in \mathrm{a}$ with $R_{X} \neq \emptyset$ then $\mu(k, \cdot) \in L_{\mathrm{loc}}^{1}(\mathfrak{a}, d X)$.

b) If in addition $\operatorname{Re}\left(l_{j}\right)<0 \forall j$ then $\mu(k, \cdot) \in L^{1}(\mathfrak{a}, d X)$.

c) If $\operatorname{Re}\left(\sum_{x \in R_{X}} k_{\alpha}\right)+r k\left(R_{X}\right)>0 \quad \forall X \in \sqrt{-1}$ a with $R_{X} \neq \emptyset$ then $\mu(k, \cdot) \in L_{\text {loc }}^{1}$ $(\sqrt{-1} \mathfrak{a}, d X)$.

Here $d X$ denotes Lebesgue measure on a and $\sqrt{-1}$ a respectively.

Proof. The proof is easy and left to the reader. QED 
Corollary 2.2 Suppose $R=R_{1} \cup \ldots \cup R_{m}$ is the decomposition of $R$ into W-orbits. Put $h_{i}:=\#\left(R_{i}\right) / r k\left(R_{i}\right)$ and $k_{i}=k_{\alpha}$ for $\alpha \in R_{i}$. Under the conditions

$$
\operatorname{Re}\left(k_{i}\right)<0 \forall i, \quad \sum_{1}^{m} \operatorname{Re}\left(k_{i}\right) h_{i}+1>0
$$

all conditions of the previous proposition are satisfied.

Proof. The $R_{i}$ are of type $A, D, E$ or a direct sum of type $A_{1}$. Note $R_{X, i}:=R_{X} \cap R_{i}$ and $h_{X, i}:=\#\left(R_{X, i}\right) / r k\left(R_{X, i}\right)$. Then it is easy to verify that $h_{X, i} \leqq h_{i}$. Since $r k\left(R_{X}\right)$ $\geqq r k\left(R_{X, i}\right)$ we get (using $\left.\operatorname{Re}\left(k_{i}\right)<0 \forall i\right)$ :

$$
\begin{aligned}
& \sum_{1}^{m} \operatorname{Re}\left(k_{i}\right) h_{i}+1>0 \Rightarrow \sum_{1}^{m} \operatorname{Re}\left(k_{i}\right) h_{X, i}+1>0 \Rightarrow \\
& \sum_{1}^{m} \operatorname{Re}\left(k_{i}\right) \cdot \#\left(R_{X, i}\right)+r k\left(R_{X}\right)>0 \Rightarrow \sum_{\alpha \in R_{X}} \operatorname{Re}\left(k_{\alpha}\right)+r k\left(R_{X}\right)>0 .
\end{aligned}
$$

Hence the conditions under a) and c) are implied by 2.4. Since $\rho(k)=\sum_{1}^{m} k_{i} \rho_{i}$ with $\rho_{i}=\frac{1}{2} \sum_{\alpha \in R_{i,+}} \alpha \in c l\left(a_{+}\right)$we have $\operatorname{Re}(\rho(k)) \in-\mathfrak{a}_{+}$for $\operatorname{Re}\left(k_{i}\right)<0$. Hcnce condition b) is implied by (2.4) as well. QED

Corollary 2.3 On the domain (2.4) in $\mathbb{C}^{m}$ the functions

$$
k \mapsto \int_{\mathfrak{a}} \mu(k, X) d X
$$

and

$$
k \mapsto \int_{V=\mathrm{T} \circ: 2 \pi \sqrt{-T} Q^{\vee}} \mu(k, X) d X
$$

are holomorphic.

\section{The case $R$ of type $B C_{n}$}

Whenever the integral converges we write

$$
\begin{aligned}
L_{n}(\alpha, \beta, \gamma)= & \int_{-\infty}^{\infty} \ldots \int_{-\infty}^{\infty} \prod_{i=1}^{n}\left(\sinh ^{2} t_{i}\right)^{\alpha} \cdot \prod_{i=1}^{n}\left(\cosh ^{2} t_{i}\right)^{\beta} \\
& \cdot \prod_{i<j}\left(\sinh ^{2}\left(t_{i}-t_{j}\right) \cdot \sinh ^{2}\left(t_{i}+t_{j}\right)\right)^{\gamma} d t_{1} \ldots d t_{n}
\end{aligned}
$$

Proposition 3.1 We have

$$
L_{n}(\alpha, \beta, \gamma)=\prod_{j=1}^{n} \frac{(j \gamma) !(2 \alpha+2(j-1) \gamma) !(-\beta-(j-1) \gamma) !(-\alpha-\beta-(n+j-2) \gamma-1) !}{(\gamma) !(\alpha+(j-1) \gamma) !(-2 \beta-2(j-1) \gamma) !}
$$


Proof. The proof is analogous to the computation in [M2, p. 992-993]: Substitute $a_{i}:=\sinh ^{2} t_{i}$ in Selberg's integral (1.4), and put $x=\alpha+\frac{1}{2}, y=-\alpha-\beta-2 \gamma(n$ $-1), z=\gamma$. QED

Remark 3.2 This proposition enables one to prove Theorem 1.1 for $R$ of type $B_{n}, C_{n}$ and $D_{n}$. However these seem to be the only cases where the non-compact integral (1.7) can be reduced to the compact integral (1.6) by a simple change of variables.

\section{From compact imaginary to non-compact real}

Choose an orthonormal basis for a and write $x=\left(x_{1}, \ldots, x_{n}\right) \in \mathbb{R}^{n}$ (or $\mathbb{C}^{n}$ ) for the coordinates of a vector $X \in \mathfrak{a}$ (respectively $\mathfrak{a}_{\mathfrak{c}}$ ). Let $\tau>0$ be a positive real number. Consider the differential $n$-form

$$
\omega=\omega(k, \tau)=\prod_{\alpha \in R_{+}}\left(e^{\frac{1}{2} \alpha(X)+\tau}-e^{-\frac{1}{2} \alpha(X)-\tau}\right)^{2 k_{\alpha}} d x_{1} \wedge \ldots \wedge d x_{n}
$$

defined on the real Weyl chamber $\mathfrak{a}_{+}$. This form has a single-valued analytic continuation as a closed differential form of type $(n, 0)$ to an open neighborhood of $c l\left(a_{c,+}\right)=c l\left(a_{+}\right) \oplus \sqrt{-1} \mathfrak{a}$ (choose the branch as for the function (2.1)). If $P^{\vee}:=\{\lambda \in \mathfrak{a} ; \alpha(\lambda) \in \mathbb{Z} \forall \alpha \in R\}$ denotes the coweight lattice in $\mathfrak{a}$ then the continuation is quasi periodic with respect to $2 \pi \sqrt{-1} P^{\vee}$, i.e. for every $X \in \mathfrak{a}_{\mathfrak{c},+}$ and $\lambda \in P^{\vee}$ we have

$$
\omega(X+2 \pi \sqrt{-1} \lambda)=\prod_{\alpha \in R_{+}} q_{\alpha}^{2 \alpha(\lambda)} \cdot \omega(X)
$$

where

$$
q_{\alpha}=e^{\pi \sqrt{-1} k_{x}}
$$

Let $\left\{\lambda_{1}, \ldots, \hat{\lambda}_{n}\right\} \subset a$ be the fundamental coweights defined by $\alpha_{i}\left(\lambda_{r j}\right)=\delta_{i j}$ and consider the parallelepiped $\Omega=[0,2 \pi] \lambda_{1}+\ldots+[0,2 \pi] \lambda_{n}$ spanned by $2 \pi \lambda_{1}, \ldots, 2 \pi \lambda_{n}$.

Proposition 4.1 If $\operatorname{Re}\left(k_{\alpha}\right)<0$ for all $\alpha \in R$ then

$$
\int_{V=-\Omega} \omega(k, \tau)=\prod_{j=1}^{n}\left(1-\prod_{\alpha \in \boldsymbol{R}_{+}} q_{\alpha}^{2 \alpha\left(\lambda_{j}\right)}\right) \cdot \int_{\mathbf{a}_{+}} \omega(k, \tau)
$$

Proof. Let $M$ be a positive real number and define parallelepipeds

$$
\begin{aligned}
\mathfrak{a}_{+, j} & :=[0, M] \lambda_{1}+\ldots+[0, M] \lambda_{j} \\
\Omega^{j} & :=[0,2 \pi] \lambda_{j+1}+\ldots+[0,2 \pi] \lambda_{n}
\end{aligned}
$$


with the convention $a_{+, 0}=\Omega^{n}=\{0\}$ and $\Omega^{0}=\Omega$. Then (4.4) will clearly follow from a repeated application of the formula $(0 \leqq j \leqq n-1)$

$$
\int_{\boldsymbol{a}_{+, j}+\sqrt{-1} \Omega^{j}} \omega=\left(1-\prod_{\alpha \in R_{+}} q_{\alpha}^{2 \alpha\left(\lambda_{j+1}\right)}\right) \cdot \int_{\mathbf{a}_{+, j+1}+\sqrt{-1} \Omega^{j+1}} \omega+O\left(e^{-c \mathbf{M}}\right)
$$

where $O\left(e^{-c M}\right)$ are terms vanishing exponentially as $M$ tends to infinity.

Formula (4.5) is proved as follows. Observe that if an $n$ dimensional parallelepiped $\sigma \subset c l\left(\mathfrak{a}_{c,+}\right)$ is spanned by $n$ vectors two of which span a complex line then $\left.\omega\right|_{\sigma}=0$. Just note that $\sigma$ is contained in a complex hyperplane. Now modulo parallelepipeds spanned by $n$ vectors two of which are $M \lambda_{j+1}$ and $2 \pi \sqrt{-1} \lambda_{j+1}$ we have

$$
\begin{aligned}
& \partial\left(\mathfrak{a}_{+, j+1}+\sqrt{-1} \Omega^{j}\right) \\
& \equiv(-1)^{j+1}\left(\left(\mathfrak{a}_{+, j}+\sqrt{-1} \Omega^{j}\right)-\left(M \lambda_{j+1}+\mathfrak{a}_{+, j}+\sqrt{-1} \Omega^{j}\right)\right) \\
& \quad+(-1)^{j+2}\left(\left(\mathfrak{a}_{+, j+1}+\sqrt{-1} \Omega^{j+1}\right)-\left(2 \pi \sqrt{-1} \lambda_{j+1}+\mathfrak{a}_{+, j+1}+\sqrt{-1} \Omega^{j+1}\right)\right)
\end{aligned}
$$

Hence equation (4.5) follows from Stokes theorem, the quasi periodicity relation (4.2) and an elementary estimate. QED

Corollary 4.2 Suppose the numbers $k_{\alpha} \in \mathbb{C}$ with $k_{w \alpha}=k_{\alpha} \forall w \in W, \forall \alpha \in R$ satisfy the conditions (2.4). Then equation (4.4) holds for $\tau=0$.

Proof. By Corollary 2.2 both sides of equation (4.4) converge for $\tau=0$. Now apply the Lebesgue dominated convergence theorem. QED

\section{Evaluation of the non-compact integral}

From now on we assume that $R \subset a^{*}$ is a reduced irreducible root system. For $\alpha \in R$ and $n \in \mathbb{Z}$ let $\alpha+n$ be the affine linear function $X \mapsto \alpha(X)+n$ on $a$. The set $\widetilde{R}=\{\alpha+n ; \alpha \in R, n \in \mathbb{Z}\}$ is the affine root system associated with $R$, and elements of $\tilde{R}$ are called affine roots. For each $a \in \widetilde{R}$ let $r_{a}$ denote the orthogonal (affine) reflection in the hyperplane $L_{a}=\{X \in a ; a(X)=0\}$. The affine Weyl group $\widetilde{W}$ is the group generated by the reflections $r_{a}$ with $a \in \widetilde{R}$. The group $\widetilde{W}$ acts on $\widetilde{R}$ by the dual action: if $w \in \tilde{W}$ and $a \in \widetilde{R}$ then $w a$ is the function $X \mapsto a\left(w^{-1} X\right)$ on $a$.

Fix $R_{+} \subset R$ set of positive roots, and let $\widetilde{R}_{+}:=R_{+} \cup R+\{1,2,3, \ldots\} \subset \widetilde{R}$ be the corresponding set of positive affine roots. Let $\left\{\alpha_{1}, \ldots, \alpha_{n}\right\} \subset R_{+}$be the set of simple roots and $S=\left\{r_{1}, \ldots, r_{n}\right\}$ the Coxeter generators for $W$. If $\theta \in R_{+}$is the highest root then $a_{0}=-\theta+1 \in \widetilde{R}_{+}$is positive, and we write $r_{0} \in \tilde{W}$ for the corresponding reflection. Then $\tilde{W}$ is a Coxeter group with generators $\tilde{S}$ $=\left\{r_{0}, \ldots, r_{n}\right\}$. Let $\mathfrak{a}_{+}=\left\{X \in \mathfrak{a} ; \alpha(X)>0 \forall \alpha \in R_{+}\right\}$be the Weyl chamber corresponding to $R_{+}$, and $C=\left\{X \in \mathfrak{a} ; a(X)>0 \forall a \in \widetilde{R}_{+}\right\}$the fundamental alcove corresponding to $\widetilde{R}_{+}$.

On $\underset{W}{W}$ we have the usual length function $l(w)$ as well as the $i$-length function $\underline{l}(w)=\left(l_{i}(w)\right)_{i=1, \ldots, m}$ where $m \in\{1,2,3\}$ is the number of $\tilde{W}$-orbits in $\widetilde{R}$ (see [B], [M1]). These functions behave nicely for the restriction from $\tilde{W}$ to $W$.

Lemma 5.1 Suppose $w \in \tilde{W}, w \neq 1$ such that $w(C) \subset a_{+}$. Then there exists $r_{i} \in \tilde{S}$ with $l\left(w r_{i}\right)<l(w)$ and $w r_{i}(C) \subset a_{+}$. Moreover if one crosses the separating wall between $w r_{i}(C)$ and $w(C)$ going from $w r_{i}(C)$ to $w(C)$ one goes in the direction of a positive root in $R_{+}$. 
Proof. Choose $X \in C$ a generic point. The straight line from $w(X)$ to $X$ intersects the boundary of $w(C)$ in the wall $L_{a}$ for some $a \in \tilde{R}$. Now clearly $L_{a}$ separates $w(C)$ and $C$. Hence $l\left(r_{a} w\right)=l(w)-1$. Since $L_{a}$ is a wall of $w(C)$ we also have $r_{a} w=w r_{i}$ for some $i$. This proves the first statement. The second statement is obvious from this construction. QED

Lemma 5.2 For $w \in \tilde{W}$ we have $l(w)=l(v)+l\left(v^{-1} w\right)$ where $v \in W$ is the element with $w(C) \subset v\left(\mathfrak{a}_{+}\right)$.

Proof. Choose $X \in C$ a generic point but close to the origin. The straight line from $X$ to $w(X)$ first meets the reflecting hyperplanes separating $C$ and $v(C)$ and then the ones separating $v(C)$ and $w(C)$. QED

In view of Corollary 2.3 it is no restriction to assume $k_{\alpha} \in \mathbb{R} \forall \alpha \in R$ which we will do from now on. Instead of the affine Weyl group $\tilde{W}$ with translation lattice $Q^{\vee}$ (the real realization) we will work from now on with the affine Weyl group with translation lattice $2 \pi \sqrt{-1} Q^{\vee}$ (the imaginary realization). By abuse of notation we denote this group again by $\tilde{W}$.

Lemma 5.3 Suppose $w \in \tilde{W}$ satisfies $w(2 \pi \sqrt{-1} C) \subset 2 \pi \sqrt{-1} a_{+}$. Then we have for $X \in 2 \pi \sqrt{-1} C$

$$
\frac{\delta(k, w(X))}{\mu(k, w(X))}=\left(\prod_{\alpha \in R_{+}} q_{x}\right) \cdot q^{2 \underline{l(w)}}
$$

with $q_{\alpha}=e^{\pi \sqrt{-1} k_{\alpha}}$ and $q^{2 l\left(w^{\prime}\right)}=\prod_{i=1}^{m} q_{i}^{2 l_{i}\left(w^{\prime}\right)}$ where $q_{r_{0}}=q_{\theta}\left(\theta \in R_{+}\right.$, the highest root $)$; $q_{r_{i}}=q_{\alpha_{i}}$ for $i=1, \ldots, n$, and the index $i \in\{1, \ldots, m\}$ indicates the conjugacy class of simple reflections under $\widetilde{W}$.

Proof. With induction on $l(w)$. If $l(w)=0$ then (5.1) is clear: Moving $X$ from $2 \pi C$ to $2 \pi \sqrt{-1} C$ one picks up a factor $\prod_{\alpha>0}(\sqrt{-1})^{2 k_{\alpha}}=\prod_{\alpha>0} q_{\alpha}$. If $l(w) \geqq 1$ then choose $r_{i} \in \tilde{S}$ with $l\left(w r_{i}\right)<l(w)$ and $w r_{i}(C) \subset a_{+}$as in Lemma 5.1. Going from $w r_{i}(2 \pi \sqrt{-1} C)$ to $w(2 \pi \sqrt{-1} C)$ one picks up one extra factor $q_{i}^{2}$ in $\delta$ relative to $\mu$. This proves the lemma. QED

Consider the Poincaré series $W(t)=\sum_{w \in W} t^{l(w)}$ and $\tilde{W}(t)=\sum_{w \in \tilde{W}} t^{l(w)}$ (see [M1]).

Lemma 5.4 We have

$$
\tilde{W}(t)=W(t) \cdot\left\{\sum_{w \in \tilde{W}, w(2 \pi V-1 C) \subset \sqrt{-1} a_{+}} t^{I^{(w)}}\right\}
$$

and

(5.3) $\sum_{w \in \tilde{W}, w(2 \pi V-1 C) \subset \sqrt{-1 \Omega}} t^{l(w)}=\prod_{j=1}^{n}\left(1-\prod_{\alpha \in R_{+}} t_{\alpha}^{\alpha\left(\lambda_{j}\right)}\right) \cdot\left\{\sum_{w \in \tilde{W}, w(2 \pi V-1 C) \subset V-1 \alpha_{+}} t^{l(w)}\right\}$

in the notation of the previous section.

Proof. The first statement is clear from Lemma 5.2. As observed in (4.2) the function $\delta$ is quasi periodic for the lattice $2 \pi \sqrt{-1} P^{\vee}$. Together with Lemma 5.3 we get (with $t=q^{2}$ ) 


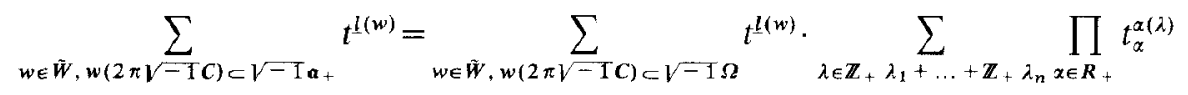

and (5.3) follows by summing the geometric series. QED

Theorem 5.5 Suppose the Lebesgue measure $d X$ on $\mathfrak{a}$ is normalized such that $\int_{a / 2 \pi Q^{\vee}} d X=1$ (this normalization is obtained from the normalized Haar measure $d t$ on $T:=\sqrt{-1} \mathfrak{a} / 2 \pi \sqrt{-1} Q^{\vee}$ by analytic continuation). If the parameter $k$ satisfies the conditions $(2.4)$ then

$$
\frac{\int_{a} \mu(k, X) d X}{\int_{T} \mu(k, t) d t}=\frac{(\sqrt{-1})^{n} \cdot \prod_{\alpha \in R_{+}} q_{\alpha} \cdot \tilde{W}\left(q^{2}\right)}{W\left(q^{2}\right)}
$$

Proof. Normalize the inner product on a such that the Lebesgue measure $d X$ on $a$ is the density associated with the differential form $d x_{1} \wedge \ldots \wedge d x_{n}$, and denote by $d Y$ the corresponding Lebesgue measure on $\sqrt{-1}$ a. Assume that $\mathfrak{a}$ is oriented such that $\int_{\Omega} d x_{1} \wedge \ldots \wedge d x_{n}=\operatorname{vol}(\Omega)$. Then we have $\int_{T} \mu(k, t) d t$

$$
\begin{aligned}
& =\int_{\sqrt{-I} \alpha / 2 \pi V-1 Q^{2}} \mu(k, Y) d Y=|W| \int_{2 \pi \sqrt{-1 C}} \mu(k, Y) d Y \\
& =\frac{|W|}{\prod_{\alpha>0} q_{\alpha} \cdot \sum_{w \in \tilde{W}, w(2 \pi V-\mathrm{T} C) \subset \sqrt{-\mathrm{T} \Omega}} q^{2 !(w)}} \cdot \int_{V-1 \Omega} \delta(k, Y) d Y \\
& =\frac{|W|}{(\sqrt{-1})^{n} \cdot \prod_{\alpha>0} q_{x^{*}} \sum_{w \in \tilde{W}, w(2 \pi \sqrt{-1} c) \subset \sqrt{-1} \Omega} q^{2 \underline{l}(w)}} \cdot \lim _{\tau \downarrow 0} \int_{V-1 \Omega} \omega(k, \tau) \\
& =\frac{|W| \cdot \prod_{j=1}^{n}\left(1-\prod_{\alpha>0} q_{\alpha}^{2 \alpha\left(\lambda_{j}\right)}\right)}{(\sqrt{-1})^{n} \cdot \prod_{\alpha>0} q_{\alpha} \cdot \sum_{w \in \tilde{W}, w(2 \pi \sqrt{-I C)} \in \sqrt{-1} \Omega} q^{2 l(w)}} \cdot \int_{a_{+}} \omega(k, 0) \quad \text { by Corollary } 4.2 \\
& =\frac{|W|}{(\sqrt{-1})^{n} \cdot \prod_{\alpha>0} q_{a} \cdot \sum_{w \in W} \sum_{w(2 \pi V-I C) \subset \sqrt{-1} a_{+}} q^{2 l(w)}} \cdot \int_{a_{+}} \mu(k, X) d X \quad \text { by (5.3) } \\
& =\frac{W\left(q^{2}\right)}{(\sqrt{-1})^{n} \cdot \prod_{\alpha>0} q_{\alpha} \cdot \tilde{W}\left(q^{2}\right)} \cdot \int_{a} \mu(k, X) d X
\end{aligned}
$$

which proves the theorem. QED

Remark 5.6 In case $k_{\alpha}=k \forall \alpha \in R$ Theorem 1.1 is easily obtained from Theorem 5.5. Indeed in this case the Poincaré series for $W$ and $\tilde{W}$ are given by

$$
W(t)=\prod_{j=1}^{n} \frac{\left(1-t^{m_{j}+1}\right)}{(1-t)}
$$


and

$$
\tilde{W}(t)=\prod_{j=1}^{n} \frac{\left(1-t^{m_{j}+1}\right)}{(1-t)\left(1-t^{m_{j}}\right)}
$$

as originally computed by Bott (see [M 1], p. 164 and p. 173). Here $m_{j}=d_{j}-1$ are the exponents of $R$. Hence the right hand side of (5.4) becomes (using $\left.\#\left(R_{+}\right)=\sum m_{j}\right)$

$$
\frac{(\sqrt{-1})^{n} \cdot q^{\# R_{+}} \cdot \tilde{W}\left(q^{2}\right)}{W\left(q^{2}\right)}=\frac{(\sqrt{-1})^{n} \cdot q_{j}^{\sum_{j} m_{j}}}{\prod_{j}\left(1-q^{2 m_{j}}\right)}=\prod_{j=1}^{n} \frac{-1}{2 \sin \left(\pi k m_{j}\right)}
$$

and Theorem 1.1 follows from (1.6) and (5.4).

\section{Final remarks}

Consider the differential operator on $A$

$$
L(k)=\sum_{j=1}^{n} \frac{\partial^{2}}{\partial x_{j}^{2}}+\sum_{x \in R_{+}} k_{\alpha} \frac{e^{\frac{1}{2} \alpha}+e^{-\frac{1}{2} \alpha}}{e^{\frac{1}{2} \alpha}-e^{-\frac{1}{2} \alpha}} \cdot \frac{\partial}{\partial \alpha}
$$

as studied in $[\mathrm{H} 0, \mathrm{H} 1,2, \mathrm{O} 1,2]$. Then there exists a commutative family $\mathbb{D}(k)$ of differential operators on $A$, depending polynomially on the parameter $k$ and containing $L(k)$, which is isomorphic to a polynomial algebra in $n$ variables. More precisely there exists an algebra isomorphism

$$
\gamma(k): \mathbb{D}(k) \rightarrow S \mathfrak{a}_{c}^{W}
$$

which can be considered as a deformation in $k$ of the Harish-Chandra isomorphism for the invariant differential operators on a Riemannian symmetric space $G / K$. In fact we have $\gamma(k)(L(k))(\lambda)=(\lambda, \lambda)-(\rho(k), \rho(k))$.

Suppose $\varepsilon>0$ small and $k_{\alpha}>-\varepsilon \forall \alpha \in R$. Then it is easy to see that the real subspace of $\mathbb{D}(k)$ corresponding to $\mathbb{R}\left[\sqrt{-1} \mathfrak{a}^{*}\right]^{W}$ under $\gamma(k)$ consists of symmetric operators for the measure $\mu(k, a) d a$ on $A$. A problem of considerable interest would be to obtain the (presumably existing) simultaneous spectral resolution of this commuting family of differential operators in explicit form. It is known that there exists a unique solution $F=F(\lambda, k ; a)$ for each $\lambda \in \mathfrak{a}_{c}^{*}$ to the system of differential equations

$$
D F=\gamma(k)(D)(i) \cdot F \quad \forall D \in \mathbb{D}(k),
$$

which is analytic and $W$-invariant on $A$ and normalized by the condition $\mathrm{F}$ $F(\lambda, k ; e)=1$. Moreover on $A_{+}$this function has an asymptotic expansion

$$
F(\lambda, k ; a) \sim \sum_{w \in W} c(w \lambda, k) a^{w \lambda-\rho(k)}
$$


with $c(\lambda, k)$ the $c$-function given by the Gindikin-Karpelevic product formula (see [O4]). In case the parameters $k_{\alpha}$ are half the root multiplicities of the restricted root system of $G / K$ then this spectral resolution is given by HarishChandra's Plancherel formula for $G / K$. It says that for $f \in C_{c}^{\infty}(A)^{W}$ we have (with $d a$ and $d \lambda$ regularly normalized)

$$
f(\cdot)=\frac{1}{|W|^{2}} \int_{\mathbf{a}^{*}}\left\{\int_{\boldsymbol{A}} f(a) F(-\sqrt{-1} \lambda, k ; a) \mu(k, a) d a\right\} F(\sqrt{-1} \lambda, k ; \cdot) \frac{d \lambda}{|c(\sqrt{-1} \lambda, k)|^{2}} .
$$

In case of rank one this formula boils down to the example of the Weyl-Titchmarsch spectral theory for the Gaussian hypergeometric function. One might expect formula (6.1) to remain valid under the assumption $k_{\alpha} \geqq 0 \forall \in R$. However the set of eigenfunctions $F(\sqrt{-1} \lambda, k ; a)$ with $\lambda \in \mathrm{a}^{*}$ fails to be complete if $k_{\alpha}$ becomes negative and small. Indeed, discrete spectrum occurs for the eigenfunction $F(\rho(k), k ; a) \equiv 1$ and what we have computed in this paper is the $L^{2}$-norm of this function.

Note that for $R$ of type $B C_{n}$ (with three root multiplicities $k_{l}, k_{m}, k_{s}$ for the long, medium and short roots) more discrete spectrum will occur when $k_{l} \ll 0$ and $k_{l}+k_{s}, k_{m}>-\varepsilon$. The eigenfunctions are analytic continuations of the multivariable Jacobi polynomials associated with $R$, and their $L^{2}$-norms can be computed by using either the contiguity relations as in [H1, Sect. 8] or the hypergeometric shift operators from [O1,2, H2]. The fact that a finite number of discrete eigenfunctions arise this way was already observed in the rank one case in [We, p. 235].

\section{References}

[B] Bourbaki, N.: Groupes et Algèbres de Lie. Ch. 4, 5 et 6, Paris: Masson 1981

[D] Dyson, F.J.: Statistical theory of the energy levels of complex systems I. J. Math. Phys. 3, 140-156 (1962)

[G] Gunson, J.: Proof of a conjecture of Dyson in the statistical theory of energy levels. J. Math. Phys. 3, 752-753 (1962)

[HO] Heckman, G.J., Opdam, E.M.: Root systems and hypergeometric functions I. Comp. Math. 64, 329-352 (1987)

[H1] Heckman, G.J.: Root systems and hypergeometric functions II. Comp. Math. 64, 353373 (1987)

[H2] Heckman, G.J.: An elementary approach to the hypergeometric shift operators of Opdam. Invent. Math. 103, 341-350 (1991)

[M1] Macdonald, I.G.: The Poincaré series of a Coxeter group. Math. Ann. 199, 161-174 (1972)

[M 2] Macdonald, I.G.: Some conjectures for root systems. Siam J. Math. Anal. 13 (6) 988 1007 (1982)

[O 1] Opdam, E.M.: Root systems and hypergeometric functions III. Comp. Math. 67, 21-49(1988)

[O2] Opdam, E.M.: Root systems and hypergeometric functions IV. Comp. Math. 67, 191209 (1988)

[O3] Opdam, E.M.: Some applications of hypergeometric shift operators. Invent. Math. 98, $1-18(1989)$

[O4] Opdam, E.M.: A Gauss summation formula for root systems (in preparation)

[S] Selberg, A.: Bemerkninger om et multipelt integral. Norsk Mat. Tidsskr. 26, 71-78 (1944) (=Coll. Papers, Vol. 1, 204-213)

[We] Weyl, H.: Über gewöhnliche lineare Differentialgleichungen mit singulären Stellen und ihre Eigenfunktionen, Nachrichten der Königlichen Gesellschaft der Wissenschaften zu Göttingen. Mathematisch-physikalische Klasse, 442-467 (1910) (=Coll. Papers, Vol. 1, 222-247)

[Wi] Wilson, K.: Proof of a conjecture by Dyson. J. Math. Phys. 3, $1040-1043$ (1962) 